\title{
Estimates of the Speed of Convergence of Continued Fraction Expansions of Functions*
}

\begin{abstract}
By David A. Field
Abstract. The best recently published methods of obtaining a priori and a posteriori truncation error estimates of continued fractions are reviewed. The applicability of these methods is discussed and a numerical example with tables of their actual estimates is provided.
\end{abstract}

1. Introduction. In 1967 Blanch [4] published her well-known paper "Numerical evaluation of continued fractions". An important aspect of her paper was estimating the speed of convergence of continued fractions. Since then many articles have been published on estimating the speed of convergence, giving a priori error estimates [6] , [7] , [12], [18], [19], a posteriori error estimates [3] , [7], $[10],[11],[13]$, and asymptotic error estimates [5], [14]. This paper compares the best a priori and a posteriori methods by presenting a Brief account of their usefulness and idiosyncracies in applications, and a comparison of numerical estimates. In order to better assess their relative merits, these methods are all applied to the same continued fraction Blanch used. A goal of this paper is to provide enough qualitative and quantitative information to enable one to make efficient choices among these methods without laboriously applying each method.

2. Error Estimate Methods. The dilemma of deciding which truncation estimate method to employ is facilitated by Table 1 . The table includes the most useful papers and omits those which have narrow applications or for which improvements have been published. Since these papers are easily obtained, pertinent definitions and terminology will be omitted here. Good general references are Wall [21], Perron [17], and Thron [20].

The choice of employing an a posteriori or an a priori estimate should depend on the information desired. In general, a posteriori methods are more easily applied but require more computer time whenever estimates are required at more than a few points. These error estimates are of the form

$$
\left|f-f_{n}\right| \leqslant M_{n}\left|f_{n}-f_{n-1}\right|,
$$

where $f$ is the value and $f_{n}$ is the $n$th approximant of the continued fraction. On the other hand, a priori estimates normally yield estimates which are uniform over some

Received June $21,1976$.

AMS (MOS) subject classifications (1970). Primary 30A22, 41A25; Secondary 41-02.

Key words and phrases. Continued fraction, truncation error, a priori, a posteriori.

* This research was supported by the United States Air Force through the Air Force Office of Scientific Research under Grant No. AFOSR-70-1888. 
region in the complex plane. A priori estimates are usually expressed in terms of the elements or parameters associated with the elements of the continued fraction. Both methods are easily coded for computerized calculations.

\section{A Priori Methods}

\begin{tabular}{|c|c|c|c|}
\hline Hayden: & $\begin{array}{l}\text { Critical points and tails } \\
\text { of continued fractions }\end{array}$ & 1965 & $\begin{array}{l}{[9, \text { p. } 296 \text { last line }} \\
\text { and p. } 301 \text { line }(6.10)]\end{array}$ \\
\hline Hayden: & $\begin{array}{l}\text { Contraction mappings and } \\
\text { tails of continued fractions }\end{array}$ & 1965 & {$[9$, Theorem 6.2] } \\
\hline Gragg: & $\begin{array}{l}\text { Series of Stieltjes and } \\
\pi \text {-fractions }\end{array}$ & 1968 & [7, Theorem 3] \\
\hline Jones-Snell: & $\begin{array}{l}\text { Parabolic element } \\
\text { regions for } K\left(a_{n} / 1\right)\end{array}$ & 1969 & {$[12$, Theorem 2.1] } \\
\hline Field-Jones: & $\begin{array}{l}\text { Complements of disk } \\
\text { element regions for } \\
K\left(1 / b_{n}\right)\end{array}$ & 1972 & [6, Theorem 2.1] \\
\hline
\end{tabular}

A Posteriori Methods

$\begin{array}{llcc}\text { Hayden: } & \begin{array}{l}\text { Critical points and } \\ \text { tails of continued } \\ \text { fractions }\end{array} & 1965 & {[9, \text { p. } 296 \text { last line] }} \\ \text { Merkes: } & \begin{array}{l}\text { Chain sequences and } \\ \text { Euler's algorithm }\end{array} & 1966 & \text { [15, Theorem 2] } \\ \text { Gragg: } & \begin{array}{l}\text { Series of Stieltjes and } \\ \pi \text {-fractions }\end{array} & 1968 & {[7, \text { Theorem 3] }} \\ \text { Jones-Thron: } & \begin{array}{l}\text { Nested lens-shaped } \\ \text { regions }\end{array} & 1971 & {[13, \text { Theorem 2.1] }}\end{array}$

TABLE 1

Methods of Estimating Truncation Error

A Posteriori Estimates. The simplest method to apply is the Jones-Thron method. This method is applicable to many types of continued fractions and it is easy to verify whether or not the elements of the given continued fraction can define a prescribed set of parameters. When $\arg a_{n}=\alpha$ in $K\left(a_{n} / 1\right)$, a corollary [13, p. 699] further simplifies this method. Error estimates are not improved by using equivalence 
transformations. $M_{n}$ in (2.1) is remarkably simple.

In Hayden's a posteriori method (2.1) is an equality with $M_{n}$ expressed in terms of the remainder and critical points of the continued fraction. Although this expression for $M_{n}$ applies to all continued fractions, a significant difficulty lies in the need to estimate the remainder and critical points. Crude estimates of the remainder can be supplied by the Worpitski or Parabola Theorems. Hayden provides general theorems for estimating the critical points. When the elements $a_{n}$ or $b_{n}$ of $K\left(a_{n} / b_{n}\right)$ are functions of $z$, the above theorems on the critical points and the remainder impose severe restrictions on $z$. Estimating the value of the critical points via mathematical induction tailored to the given continued fraction expansion often relaxes these restrictions. Despite the estimates of the remainder and critical points, Hayden's error bounds can be very sharp.

The a posteriori estimates of Gragg and Merkes apply to $g$-fractions. The first step in either method is to verify that the given continued fraction is a $g$-fraction. If the theoretical considerations, such as the theory of series of Stieltjes, does not provide an immediate answer, mathematical induction on the elements of the continued fraction may be helpful.

Gragg's method begins by constructing another continued fraction, $\pi$-fraction, whose even part is the original $g$-fraction. The error estimate is then given in the form (2.1) where $f_{n-1}$ is replaced by the $(2 n-1)$ th approximant of the $\pi$-fraction. $M_{n}$ in (2.1) is similar to the Jones-Thron $M_{n}$. The need to compute the $(2 n-1)$ th approximant of another continued fraction makes the calculation of error estimates require significantly greater amounts of computer time. Gragg's error bounds offer the important advantage of being easily obtained near cuts and poles in the convergence regions of the $g$-fractions. These error bounds are "best" in the sense that they measure the diameter of open convex regions consisting of the $(n+1)$ th approximants of all $g$-fractions and $(2 n+1)$ th approximants of the corresponding $\pi$-fractions where the $g$-fraction's first $n$ elements agree with the given $g$-fraction.

In the Merkes method it is necessary to construct a chain sequence. Fortunately, it suffices to construct a maximal chain sequence, see Wall [21, p. 81]. It is also necessary to estimate quantities, $\rho_{n}$, which are closely related to Hayden's critical points. An algorithm due to Euler provides these estimates. Constructing a maximal chain sequence and estimating $\rho_{n}$ impose severe restrictions on the elements of $K\left(a_{n} / b_{n}\right)$. On the other hand, error estimates are very sharp. Blanch's estimates are special cases in Merkes' paper.

A Priori Estimates. Of the a priori estimates given in the papers listed in Table 1 the easiest to evaluate is Gragg's which is of the form

$$
K(z)|(1-\sqrt{1+z}) /(1+\sqrt{1+z})|^{n-1},
$$

with $z$ suitably normalized. When $z$ is near a pole or a cut in the domain of convergence of the $g$-fraction, this estimate is not sharp. As with Gragg's a posteriori estimates, consideration must be given to verifying that one has a $g$-fraction and finding its domain of convergence. 
The Jones-Snell and Field-Jones methods yield estimates that are given in terms of the parameters which define regions locating the elements of the continued fraction. Both estimates can be applied to many types of continued fractions.

The truncation error bounds of Jones-Snell can be vastly improved if in their original main theorem the constant $M$ is replaced by $M_{n}=\left|a_{n}\right| /\left(\rho_{n} \rho_{n-1}\right)$. The adjustment requires a slight modification in their proof. The best estimates are obtained by choosing parameters to define parabolic regions with the numerator elements of $K\left(a_{n} / 1\right)$ on the axis of the parabolas. This allows the advantage of letting the parameter $k$ equal zero.

The Field-Jones method requires choosing parameters which define sequences of circles. The best choices of these parameters are not obvious. However, in wellbehaved situations, lemmas, such as their Lemma 3.1, quickly resolve this difficulty. The error estimates are very sensitive to the parameter $\delta_{n}$. The other parameters can vary a lot for the same value of $\delta_{n}$ and produce negligible differences in the error estimate. This method is the most useful method of determining truncation error estimates for continued fractions of the form $K\left(1 / b_{n}\right)$.

Hayden's critical point a priori estimates are derived from his a posteriori estimates. $\left|f_{n}-f_{n-1}\right|$ in $(2.1)$ is replaced by a product whose terms are defined by the elements and the critical points of the continued fraction. Even though each term of the product requires an estimate of a critical point, the final error bound is remarkably sharp.

Hayden's contraction mapping method applies to all continued fractions. In this method the error estimate is a product of Lipschitz constants whose values involve the elements and remainders of the continued fraction. In order to estimate the constants and remainders, restrictions similar to the restrictions in Hayden's other methods are imposed on the elements of the continued fraction. The error estimates may not be as sharp as Hayden's other error estimates.

3. Numerical Comparisons. The error estimate methods in Table 1 were applied to the ratio of successive Bessel functions of integral order and evaluated for $m=1$. The tables of numerical values of these estimates were calculated on a CDC 6400 with double precision arithmetic. Tables 2 and 3 list the error estimates determined by the methods in Table 1. For those estimates whose calculations involve the argument $z$, both the best and the worst estimate are given for $z=e^{i \theta}, \theta=0^{\circ}\left(1^{\circ}\right) 90^{\circ}$. Table 2 also indicates the region where the error bound is uniform. Only the first quadrant in the complex plane is considered, since for $z \neq 0$

$$
\frac{J_{1}(z)}{J_{0}(z)}=-\frac{J_{1}(-z)}{J_{0}(-z)} \text { and } \frac{J_{1}(\bar{z})}{J_{0}(\bar{z})}=\overline{\left(\frac{J_{1}(z)}{J_{0}(z)}\right)} \text {. }
$$

More information concerning the calculation of Bessel Functions and their ratios is contained in [1], [2], [4], [16].

The Jones-Snell estimates were substantially improved by using $M_{n}$ as suggested in Section 2. An improvement of Gragg's estimates by using $\sec (\theta-\pi / 2)$ rather than $\tan \theta / 2$ for $\pi / 2<\theta<\pi$ was negligible. 


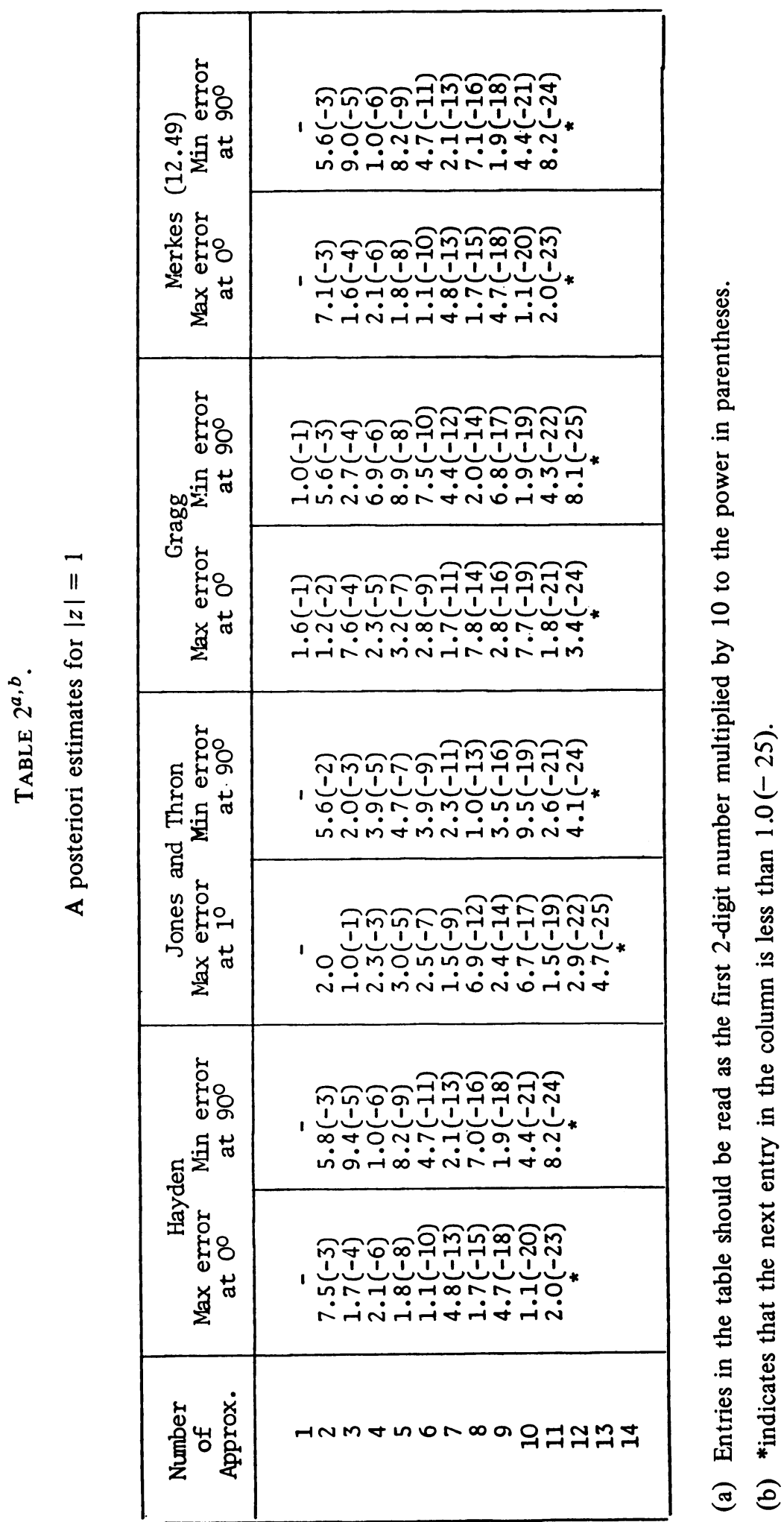




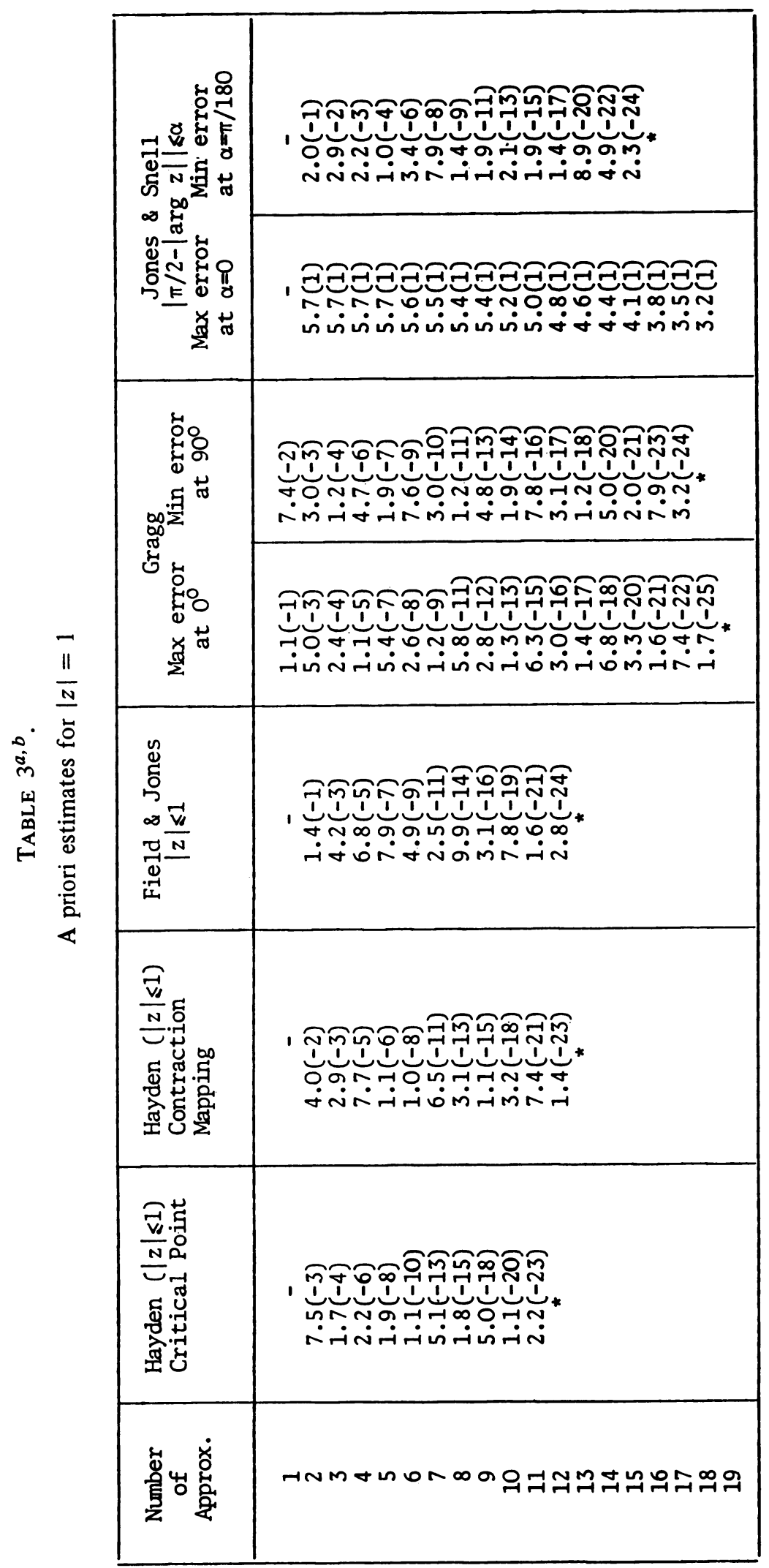



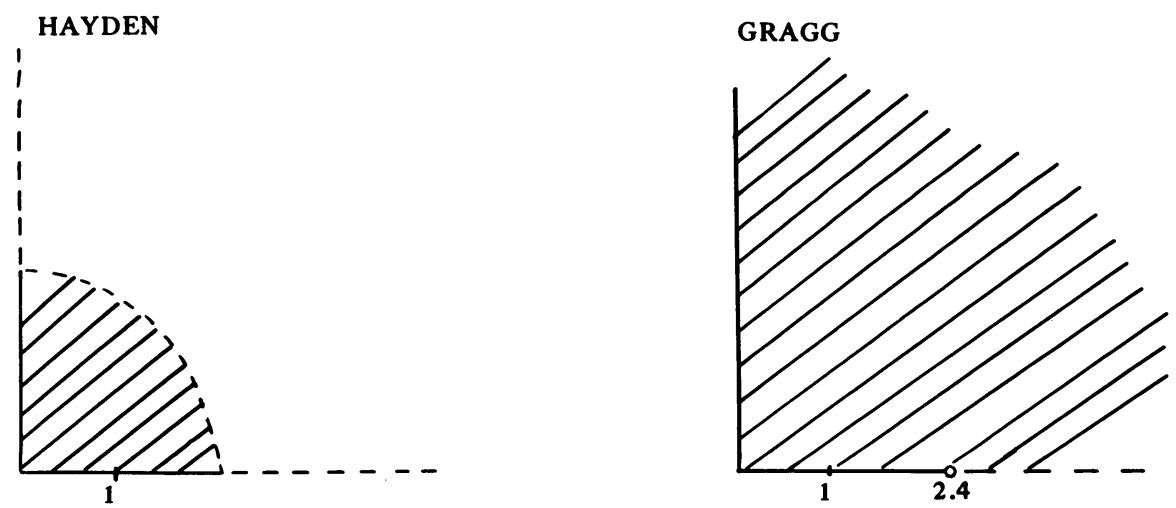

FIELD AND JONES

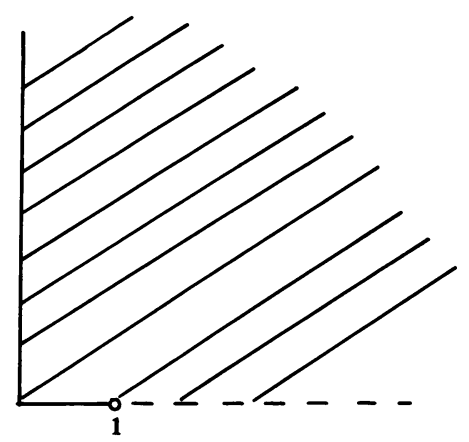

JONES AND SNELL

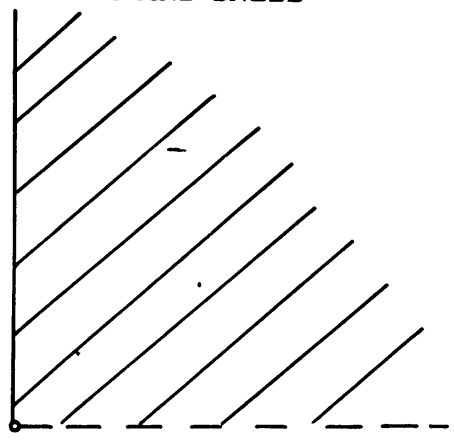

JONES AND THRON

MERKES
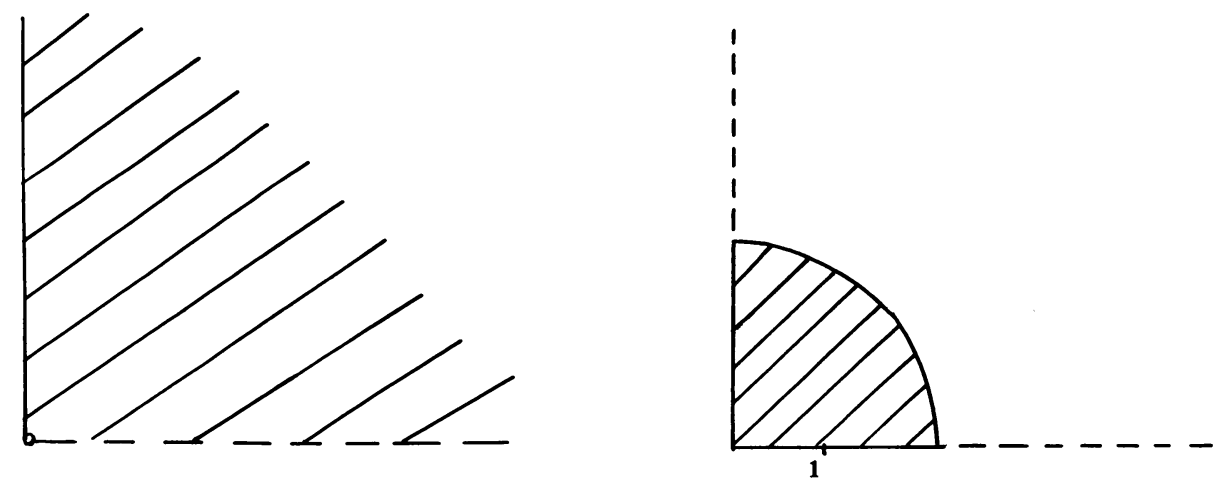

This section concludes with graphs indicating the regions of applicability of these methods over the first quadrant. It is interesting to note the restrictions away from the real axis and the first nonzero pole of $J_{1}(z) / J_{0}(z)$ at approximately 2.4 .

Department of Mathematics

College of the Holy Cross

Worcester, Massachusetts 01610

1. M. ABRAMOWITZ \& I. A. STEGUN (Editors), Handbook of Mathematical Functions, With Formulas, Graphs and Mathematical Tables, Nat. Bur. Standards, Appl. Math. Ser., no. 55, 
Superintendent of Documents, U.S. Government Printing Office, Washington, D.C., 1966. MR 34 \#8607.

2. D. E. AMOS, "Computation of modified Bessel functions and their ratios," Math. Comp., v. 28, 1974, pp. 239-251. MR 48 \#11612.

3. G. A. BAKER, JR., "Best error bounds for Padé approximants to convergent series of Stieltjes," J. Mathematical Phys., v. 10, 1969, pp. 814-820. MR 41 \#3722.

4. G. BLANCH, "Numerical evaluation of continued fractions," SIAM Rev., v. 6, 1964, pp. 383-421. MR 30 \#1605.

5. D. ELLIOTT, "Truncation errors in Pade' approximations to certain functions: An alternative approach," Math. Comp., v. 21, 1967, pp. 398-406. MR 37 \#3252.

6. D. A. FIELD \& W. B. JONES, "A priori estimates for truncation error of continued fractions $K\left(1 / b_{n}\right), "$ Numer. Math., v. 19, 1972, pp. 283-302. MR 47 \#2799.

7. W. B. GRAGG, "Truncation error bounds for $g$-fractions," Numer. Math., v. 11, 1968, pp. 370-379. MR $37 \# 3742$.

8. W. G. GRAGG, "Truncation error bounds for $\pi$-fractions," Bull. Amer. Math. Soc., v. 76, 1970, pp. 1091-1094. MR 41 \#328.

9. T. L. HAYDEN, "Continued fraction approximation to functions," Numer. Math., v. 7, 1965, pp. 292-309. MR $32 \# 3258$.

10. P. HENRICI \& P. PFLUGER, “Truncation error estimates for Stieltjes' fractions," Numer. Math., v. 9, 1966, pp. 120-138. MR 35 \#3856.

11. T. H. JEFFERSON, "Truncation error estimates for T-fractions," SIAM J. Numer. Anal., v. 6, 1969, pp. 359-364. MR $41 \# 4775$.

12. WILLIAM B. JONES \& R. I. SNELL, "Truncation errors bounds for continued fractions," SIAM J. Numer. Anal., v. 6, 1969, pp. 210-221. MR 40 \#1000.

13. WILLIAM B. JONES \& W. J. THRON, "A posteriori bounds for the truncation error of continued fractions," STAM J. Numer. Anal., v. 8, 1971, pp. 693-705. MR 45 \#602.

14. Y. L. LUKE, "The Padé table and the $\tau$-method," J. Math. Phys., v. 37, 1958, pp. 110-127. MR 20 \#5558.

15. E. P. MERKES, "On truncation errors for continued fraction computations," SIAM J. Numer. Anal., v. 3, 1966, pp. 486-496. MR 34 \#2156.

16. M. ONOE, Tables of Modified Quotients of Bessel Functions of the First Kind for Real and Imaginary Arguments, Columbia Univ. Press, New York, 1958. MR 20 \#2092.

17. O. PERRON, Die Lehre von den Kettenbruchen, 2nd ed., Teubner, Leipzig, 1929; photographic reprint, Chelsea, New York, 1950. MR 12, 254.

18. W. B. SWEEZY \& W. J. THRON, "Estimates of the speed of convergence of certain continued fractions," SIAM J. Numer. Anal., v. 4, 1967, pp. 254-270. MR 35 \#763.

19. W. J. THRON, “On parabolic convergence regions for continued fractions," Math. $Z$., v. 69, 1958, pp. 173-182. MR 20 2562.

20. W. J. THRON, “A survey of recent convergence results for continued fractions," Rocky Mountain J. Math., v. 4, 1974, pp. 273-282. MR 50 \#2467.

21. H. S. WALL, Analytic Theory of Continued Fractions, Van Nostrand, New York, 1948. MR 10, 32.

22. G. N. WATSON, A Treatise on the Theory of Bessel Functions, 2nd ed., Cambridge Univ. Press, London; Macmillan, New York, 1944. MR 6, 64. 